\title{
Measurement of Lower Limb Joint Kinematics using Inertial Sensors During Stair Ascent and Descent in Healthy Older Adults and Stroke Survivors
}

\author{
Annemarie Laudanski ${ }^{1}$, Brenda Brouwer ${ }^{2}$ and Qingguo $\mathbf{L i}^{1 *}$ \\ ${ }^{1}$ Department of Mechanical and Materials Engineering, \\ ${ }^{2}$ School of Rehabilitation Therapy, Queen's University, Kingston, ON, Canada
}

Submitted May 2013. Accepted for publication September 2013.

\begin{abstract}
This study validated the feasibility of inertial sensors in estimating lower limb joint kinematics during stair ambulation in healthy older adults and stroke survivors. Three dimensional motion data were collected using an inertial sensor-based system from 9 persons with stroke and 9 healthy older adults as they ascended and descended a staircase at a self-selected pace. The measured joint angles were compared with a laboratory-based motion capture system by computing differences in range of motion (RoM), grand mean error, standard deviation, and coefficients of multiple correlations. For stroke survivors, differences in RoM measurements between these two systems were determined to be $3.3 \pm 8.1^{\circ}$, while the highest correlations were found in the estimation of sagittal plane joint angles after offset correction. Results suggest that the inertial sensor system is suitable for estimating major joint angles in healthy older adults as well as the RoM for stroke survivors. New calibration procedures are necessary for applying the technology to a stroke population.
\end{abstract}

Keywords: stroke, inertial measurement units, gait analysis, joint kinematics, stair ambulation

\section{INTRODUCTION}

Stair negotiation (ascent and descent) is among the most demanding activities of daily living and an essential skill for independent ambulation, with difficulty reported by one third of community-dwelling older adults $[1,2]$. Compared to level ground walking, stair ascent and descent have been shown to be significantly more demanding biomechanical and neuromuscular tasks [3-5], and as a result, aging individuals employ adaptation techniques in stair negotiation as their strength and joint mobility decline [6]. For those with limited mobility, such as individuals who have suffered a stroke, the impact of residual impairments in strength, movement coordination, and balance may become more pronounced with increased task demands, thus limiting the independence of these individuals. Strong evidence suggests that the ability to negotiate stairs is

*Corresponding author: Qingguo Li, Department of Mechanical and Materials Engineering, Queen's University, 130 Stuart street, Kingston, ON K7L 3N6, Canada. Phone: (613)-533-3191. Fax: (613)-533-6489. E-mail: qli@me.queensu.ca. Other authors: a.laudanski@queensu.ca; brouwerb@queensu.ca. 
dependent on adequate lower limb strength $[7,8]$. Although $60-70 \%$ of stroke survivors regain the ability to walk, only $7-22 \%$ are able to walk independently outside their homes[9-11] and it has been shown that the best predictor of independent activity in community-dwelling individuals is the performance of stair negotiation [12-14].

In clinical settings, community ambulation capability is conventionally estimated using standard tests in lieu of real world mobility activities [9, 15]. Quantitative threedimensional gait analysis is a widely used tool in research and clinical settings for the measurement of lower-limb joint kinematics in both healthy and impaired individuals. However, although accurate, the current laboratory-based optoelectronic movement analysis has limited applicability due to the complexity of marker placement and data collection protocols, as well as the restricted measurement volume attributable to the required line of sight between cameras and markers. In addition, subjects are known to modify their gait patterns in laboratory settings, resulting in over performance compared to their day-to-day walking capability [16-18].

The growing need to study gait outside a laboratory setting has led to the development of portable inertial-sensor-based systems for gait assessment. Inertial measurement units (IMUs) composed of 3D accelerometers, gyroscopes, and magnetometers, are commercially available, relatively low-cost, and provide a portable means of acquiring spatio-temporal gait information. Through sensor fusion algorithms such as Kalman filtering, the sensor signals (measures of angular velocity, acceleration, and earth's magnetic field) can be combined to estimate the 3D orientation of each IMU's coordinate system (CS) with respect to a global CS [19-22]. Using these sensors, gait analysis could be performed in a natural setting to estimate joint kinematics given that an IMU is attached to each body segment of interest, an anatomical CS is defined for each body segment, and the orientations of the anatomical CSs are expressed in the IMU CSs [16].

Thus far, IMUs have been used to measure 3D body segment orientations in both healthy and impaired individuals [16, 23-28]. However, the definition of anatomical CSs remains critical. The inherent properties of IMUs are such that no information can be provided about the position of each individual sensing unit relative to segment bony landmarks, and therefore accurate calibration is required in order to locate sensor positions and orientations relative to anatomical references. Different calibration techniques have been conceived to ensure suitability of IMU measurements for gait analysis. O'Donovan et al. [23] defined a protocol for the 3D inter-segment ankle joint measurement, where calibration involved a novel two-stage technique in which subjects performed a rotation of both foot and leg segments about the longitudinal axis of the body while standing followed by a knee extension, maintaining alignment of the mediolateral axes of both foot and lower leg, about the respective $\mathrm{z}$-axes while seated. These motions, although suitable for healthy individuals, may not be easily performed by some clinical populations, e.g., chronic stroke survivors.

Picerno et al. [24] proposed a protocol for estimating lower limb joint kinematics by defining anatomical CSs through direct measurement of directional anatomical axes using palpable anatomical landmarks. For each body segment, the determination of the orientation of two or more non-parallel lines was required, which in turn involved using 
multiple calibration tasks and specialized equipment. This protocol increased the complexity and duration of the IMU calibration procedure.

Cutti et al. [16] proposed the estimation of lower limb joint angles through a novel protocol, named 'Outwalk'. In this protocol, two calibration procedures were performed: the mean knee joint flexion-extension axis was first computed in the thigh embedded CSs of the IMUs, followed by the definition of anatomical/functional CSs for the determination of each individual segment orientation in the IMU CS of their corresponding segment. Both calibration procedures were performed either upright or supine, and movements were self-initiated or aided by a therapist as necessary. From the calibration procedures, joint angles for the lower limbs were computed. The Outwalk protocol has since been validated with healthy subjects [29] as well as in the assessment of children with cerebral palsy [26]; both studies reported the protocol in combination with IMUs to be suitable for gait analysis in non-laboratory settings. Although applicable to clinical populations, the Outwalk protocol requires the completion of assisted calibration procedures which add a level of complexity to the data collection.

The estimation of lower limb kinematics using IMUs can be assessed through acceptable ranges of variation in lower limb joint angle estimations reported in previous studies using traditional motion capture. Yavuzer et al. reported that in individuals having suffered a stroke, angular kinematics of both affected and less-affected lower limb joints are extremely stable, with intraclass correlation coefficients (ICCs) always greater than 0.90 [30]. Between repeated measures taken 2 weeks apart on stroke subject's affected limbs, maximum differences of $2.9 \mathrm{deg}$ at the ankle, $4.9 \mathrm{deg}$ at the knee and $3.3 \mathrm{deg}$ at the hip were measured during gait by Novak et al. [31]. Significant differences in estimates between groups (control and stroke) or between limbs (affected and less affected in stroke) have been reported as a difference greater or equal to $4 \mathrm{deg}$. [32]. Therefore, error between lower limb joint angles estimated using IMUs and motion capture must fall within these acceptable ranges for IMU kinematic measures to be considered reliable.

The objective of this study was to validate the feasibility of IMUs in measuring lower-limb joint angles of chronic stroke survivors during stair ambulation. The application of IMUs, in previous studies using various calibration protocols, has been focused on gait analysis of overground walking in both healthy and impaired ambulators [26, 29, 33]. However, there remains a lack of knowledge as to the performance of these inertial sensors in other activities such as stair ambulation. This study is the first to use IMUs in the assessment of stroke survivors during stair ambulation, an important activity of daily living for independent ambulators.

\section{METHODOLOGY}

\subsection{Subjects}

Ten chronic, hemiparetic stroke survivors (a minimum of 6 months post-stroke) were recruited from the community through newspaper advertisements and letters and flyers sent to patients who attended monthly hospital-based rehabilitation programs during the past $12-18$ months. The five male and five female subjects post-stroke were $69.7 \pm 9.5$ 
of age; $1.65 \pm 0.09 \mathrm{~m}$ in height; weighed $70.47 \pm 15.05 \mathrm{~kg}$; and were $6.5 \pm 5.4$ years poststroke. Ten age- and sex-matched control subjects were also recruited through newspaper advertisements and postings at the local senior's center (age: $64.9 \pm 11.5$ years; height: $1.74 \pm 0.13 \mathrm{~m}$; weight: $80.72 \pm 15.01 \mathrm{~kg}$ ). Due to limitations in one subject's stair ambulation ability, only 9 from each group were included in the analysis. All healthy and post-stroke subjects self-reported to be in good health and were willing to participate in an orientation session and approximately 3 hours of testing. In addition, subjects were required to be able to ascend and descend at least 4 steps, with or without the use of a handrail. Subjects with stroke were screened to ensure that they were independent ambulators, with or without the use of a cane or assistive device, had a unilateral mobility deficit, and were able to follow instructions. All subjects were screened to exclude those with a history of non-stroke related mobility restrictions (neurological and musculoskeletal), cardiac disease (unstable angina, peripheral vascular disease, congestive heart failure), uncontrolled hypertension (systolic pressure $>140 \mathrm{mmHg}$, or diastolic pressure $>90 \mathrm{mmHg}$ ), or cognitive deficits. All subjects gave their informed consent prior to participation in accordance with university policy, and the study was approved by the Queen's Health Science Research Ethics Board (HSREB).

\subsection{Instrumentation}

The IMU system used in this study was the Xsens MVN Biomech system (Xsens Technology B.V., Netherlands) which was composed of 7 inertial sensors. Sensors were attached to the lateral aspect of each subject's midthigh and midshank, as well as the superior aspect of the midfoot, with an additional inertial sensor positioned over the pelvis, at the level of the L5S1 joint, using customized elastic straps (Xsens Technologies B.V., Netherlands) as seen in Figure 1. Data were collected at a sampling frequency of $120 \mathrm{~Hz}$.

Calibration of the IMU sensors and data collection was conducted using MVN Studio Pro V.3.0 (Xsens Technologies B.V., Netherlands). The calibration of these IMUs was conducted prior to commencing stair trials and allowed for the expression of segment kinematics within the global frame by determining the orientation of each sensor module with respect to the limb on which they were attached, as well as the relative distances between sensors. In order to define the sensor to segment alignment, subjects were asked to stand in a neutral position or N-pose (feet parallel, one foot width apart; legs and back straight; arms straight down at their sides; palms facing the body). The sensor to segment rotations were determined by matching the orientation of each sensor in the global frame with a known orientation of each segment (the known orientation is defined assuming the individual has adopted a perfect N-pose) [34]. Within MVN Studio, several checks were performed on the sensor signals to determine the quality of the calibration, including a measure of standing still, the expected alignment of the pelvis IMU sensor, and the homogeneity of the magnetic field in the surrounding area (Xsens Technology B.V., Netherlands). The software then reported calibration quality as Good, Acceptable, Fair or Poor based on body pose and sensor locations, and a minimum of Acceptable quality was ensured for each subject calibration. The rotation angle between the sensor and the body segment was then 


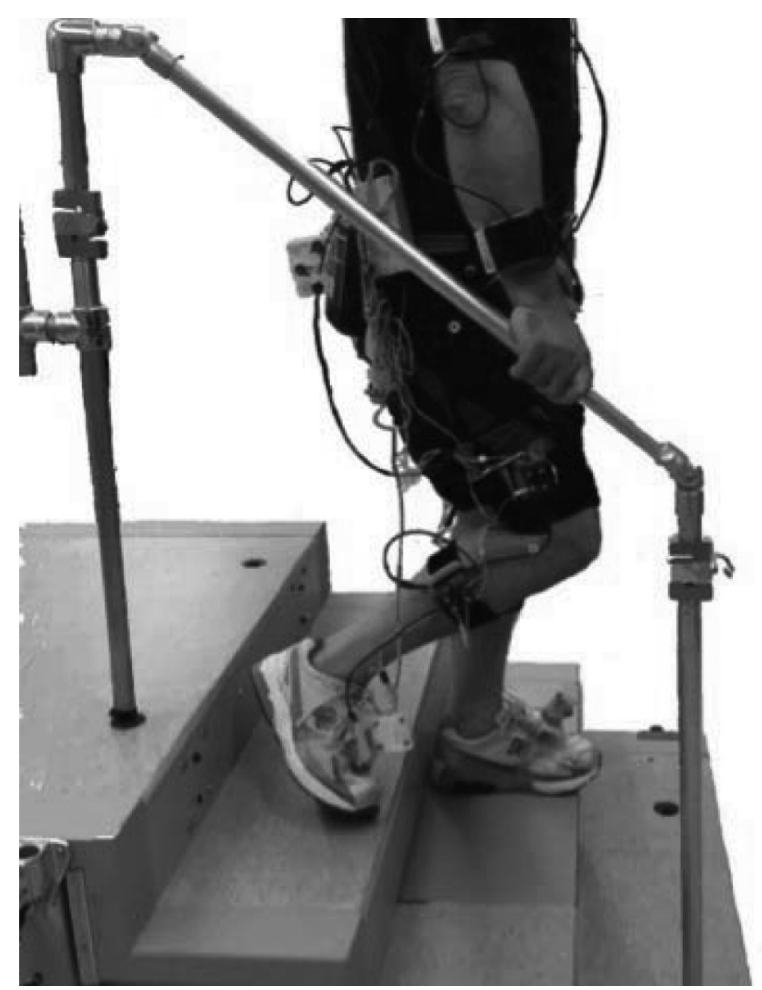

Figure 1. A subject instrumented with IMU and IRED sensors descending the instrumented staircase onto the force platform.

determined within MVN Studio by comparing the orientation of the sensor in the global frame with the nominal orientations of each body segment within this pose (assumed based on the known orientation of each limb segment in the perfectly held N-pose) [34]. Estimates of joint positions were obtained through the measurement of several anthropometric body dimensions and anatomical references including: body height, foot size, ankle height (ground to distal tip of lateral malleolus), knee height (ground to lateral epicondyle on the femoral bone), hip height (ground to most lateral bony prominence of greater trochanter), and hip width (right to left anterior superior iliac spine).

The optoelectric motion capture system was composed of two optoelectric cameras (Optotrak 3020, Northern Digital Inc., Waterloo, ON) positioned on either side of the staircase, which were used to track the position of individual infrared emitting diodes (IREDs) (accuracy up to $0.1 \mathrm{~mm}$, resolution $0.01 \mathrm{~mm}$ ). These IREDs were mounted non-collinearly in clusters of three to four to each IMU positioned on subjects' lower limbs using double sided tape. IREDs were also positioned over the sacrum at the level of S2 using an outward projecting fin of known dimensions. Data were acquired at a sampling frequency of $50 \mathrm{~Hz}$. 
Calibration of the optoelectric system was conducted after subjects completed stair ascent and descent trials, using a probe embedded with four IREDs fixed at known orientation to the tip. While instrumented, subjects stood on the second stair and the first and fifth metatarsal heads, medial and lateral malleoli, medial and lateral epicondyles, greater trochanters and the anterior superior iliac spines were probed thereby defining virtual landmarks $[35,36]$. These data enabled the definition of limb segment lengths, joint centers, and the rotational axes of each limb segment within NDI ToolBench $^{\mathrm{TM}}$ (Northern Digital Inc., Waterloo, ON).

All experiments were conducted using an instrumented staircase consisting of four steps (rise: $15 \mathrm{~cm}$; run: $26 \mathrm{~cm}$; width: $56 \mathrm{~cm}$ ). A force platform (AMTI, Newton, MA) mounted on concrete blocks replaced the middle portion of the second step and recorded ground reaction forces at a sampling frequency of $100 \mathrm{~Hz}$.

\subsection{Procedure}

Once instrumented, subjects were asked to ascend and descend the staircase with lead and connecting wires well secured and ensuring no obstruction of the IREDs or restriction of subjects' ability to ambulate naturally. Subjects were instructed to perform trials at a self-selected pace either using the step-over-step (SOS) method placing one foot on each stair, or the step-by-step (SBS) method placing both feet on the same stair led by the dominant/ less-affected foot. Subjects were asked to pause between each trial, and when they indicated that they were ready to continue, they were given a 'go' command and commenced the next trials. Trials were performed with $(\mathrm{H})$ and without $(\mathrm{NH})$ the use of a handrail on subjects' dominant side. For each subject, data of three successful trials were acquired for each condition (SOS H, SOS NH, SBS H, SBS NH) for both ascent and descent. Only the results for the SOS H conditions were reported in this paper. The results for other trial conditions (SOS NH, SBS H, SBS NH) are similar to the SOS H conditions.

\subsection{Measurement of Joint Angles}

Using a 9-segment biomechanical model of the lower body (pelvis, upper leg, lower leg, foot, and toe), the IMU kinematic data were transformed into body segment kinematics within MVN Studio Pro. The orientation and position of each sensor were calculated in the global frame, respectively, using integration of the gyroscope measurements and double-integration of the accelerometer readings. After integration of these signals, a Kalman filter was applied to correct for orientation and rotation errors in calculations. Magnetometer data were employed in the correction of rotation errors around the vertical axis, in order to correct the drift of the accelerometer and gyroscope at each time step [34]. From these results, lower limb joint angles were determined by calculating the orientation of the distal body segment with respect to that of the proximal body segment in the global frame, where these segment orientations were determined by the quaternion multiplication of the orientation of the sensor in the global frame with the complex conjugate of the rotation from sensor to body segment. Second-order forward-backward low-pass Butterworth filters were applied to the IMU joint angle data, with a cutoff frequency of $6 \mathrm{~Hz}$ to remove noise from the raw angular 
velocity and acceleration measurements [37]. IMU data were then segmented to include only the stance phase of the involved limb in contact with the force platform due to IRED marker occlusion in late swing. Heel strike to toe off was determined manually through visual inspection of the kinematic data from the IMU signal, and stance phase was normalized to 101 data points. IMU data were processed offline using MATLAB (The MathWorks, Natick, MA, USA).

All motion capture and force platform data were filtered (second-order low-pass Butterworth, cutoff frequency $6 \mathrm{~Hz}$ ), and stance phase was normalized to 101 points and synchronized using Visual 3D (C-Motion Inc., Rockville, MD). Detection of stance phase over the force platform was determined from the gait events of heel strike and toe off within each motion trial. Lower limb joint angles were computed using a sevensegment, link-segment model where spatial coordinates were transformed into Cardan angles by determining the orientation of the distal segment with respect to the reference proximal segment using the $\mathrm{x}, \mathrm{y}, \mathrm{z}$ ordered sequence of rotations (representing flexion/extension (FE), abduction/adduction (AA), and internal/external (IE) rotation, respectively) as suggested for such subject groups by Novak and Brouwer [6, 32]. Joint angle data for the ankle, knee, and hip during stance were then exported from Visual 3D to be further analyzed. Missing marker data resulting in incomplete joint angle curves (with no more than 15 consecutive incomplete frames of data) were interpolated using the MATLAB polynomial fit function, interp1.

As the IMU and motion capture data were used to synchronously measure subjects' motion during all trials and shared the same anatomical coordinate system definitions, both systems suffered the same soft-tissue artifact. Thus, differences in kinematics should be related only to differences in the ability of each measurement system to measure joint angles and to the effects of IMU calibration on these joint angle estimation.

\subsection{Data Analysis}

In order to assess the accuracy of the IMUs' measurement of joint kinematics, joint angle data collected during the stance phase from both the IMU and motion capture protocols were compared. For each protocol, the computed FE, AA, and IE rotation angles at the ankle, knee and hip were evaluated using the following parameters: mean (mean of the absolute value of motion capture signal - IMU signal), standard deviation (SD), and standard error of the mean (SEM; $\left.\frac{S D}{\sqrt{(\text { number of IMU samples })}}\right)$ for (i) the difference in range of motion (RoM: maximal minus minimal joint angle), and (ii) the error between protocols (the grand mean error, expressed in degrees) over all subjects. These values were calculated with original joint angle data as well as after the mean difference between waveforms was subtracted to remove any offset present in IMU data between joint angle estimations due to initial calibration methods. For control subjects, only data collected from the dominant limbs were analyzed, assuming symmetry between limbs. However for stroke subjects, analysis was performed for both affected and less-affected limbs. To determine whether offset correction had a significant effect 
on the calculation of RoM, SEM, and SD, student's $t$-tests were applied to examine the significance of differences, with $\mathrm{p}<0.01$ indicating statistical significance.

The adjusted coefficients of multiple correlation $(C M C)$ were calculated between IMU and motion capture protocols for original joint angles, as well as once the mean difference between waveforms was removed, $C M C_{1}$ and $C M C_{2}$, respectively. $C M C$ is a measure of similarity of waveforms taking into account the concurrent effects of differences in offset, correlation, and gain among them. Originally, $C M C$ was proposed in two formulations, noted as within-day and between-day CMC [38]. In this study, the need to compare waveforms from different protocols requires the use of the betweenday or inter-protocol similarity to determine the variability in the measurement system performances [39]. Assuming synchronously measured kinematics through $P$ protocols on $G$ gait-cycles (each of $F_{g}$ frames), the $C M C$ formulation was calculated:

$$
C M C=\sqrt{1-\frac{\sum_{g=1}^{G}\left[\sum_{p=1}^{P} \sum_{f=1}^{F}\left(Y_{g p f}-\bar{Y}_{g f}\right)^{2} / G F_{g}(P-1)\right]}{\sum_{g=1}^{G}\left[\sum_{p=1}^{P} \sum_{f=1}^{F}\left(Y_{g p f}-\bar{Y}_{g}\right)^{2} / G\left(P F_{g}-1\right)\right]}}
$$

with the parameters defined below:

- $\quad Y_{g p f}$ : ordinate at frame $f$ of the waveform provided by protocol $p$ at gait cycle $g$.

- $\bar{Y}_{g f}$ : ordinate at frame $f$ of the average waveform among $P$ waveforms of gait-cycle $g$, such that

$$
\bar{Y}_{g f}=\frac{1}{P} \sum_{p=1}^{P} Y_{g p f} .
$$

- $\quad \bar{Y}_{g}$ : grand mean for the gait-cycle $g$ among its $P$ waveforms, such that

$$
\bar{Y}_{g}=\frac{1}{P F} \sum_{p=1}^{P} \sum_{f=1}^{F} Y_{p f} \text {. }
$$

In this study each trial contains only 1 gait-cycle. However, $C M C$ values were calculated independently for both SOS ascent and SOS descent, and for affected or lessaffected limbs (stroke) or the dominant leg only (control), ( $p=2$ protocols [IMU and motion capture], $g=3$ gait-cycles [healthy, less-affected, affected], $f=101$ frames).

Within each gait cycle, if the variability of each waveform about the mean waveform is smaller than the variance about their grand mean, the $C M C$ value approaches 1 ; otherwise, the $C M C$ value will approach zero. If the offset between curves is not removed and this offset is comparable to the RoM of these curves, the numerator of Eq. 1 may become greater than the denominator, therefore resulting in a complex number for the $C M C$ value. Based on previous studies [30, 38, 40], the $C M C$ values in this study were interpreted as follows: 
- $\quad 0.65-0.75$ : moderate

- $\quad 0.75-0.85: \operatorname{good}$

- $\quad 0.85-0.95$ : very good

- $\quad 0.95-1$ : excellent

\section{RESULTS}

Sample kinematic results estimated using IMUs and motion capture for one stroke subject during SOS stair descent is shown in Figure 2. A high level of similarity is observed between flexion extension curves, while marked differences are observed for abduction/adduction and internal/external rotation estimations.

From the motion capture system, mean range of motion values were obtained for control subjects' dominant limbs as well as stroke subjects' affected and less affected limbs. These values are exhibited in Table 1 below.
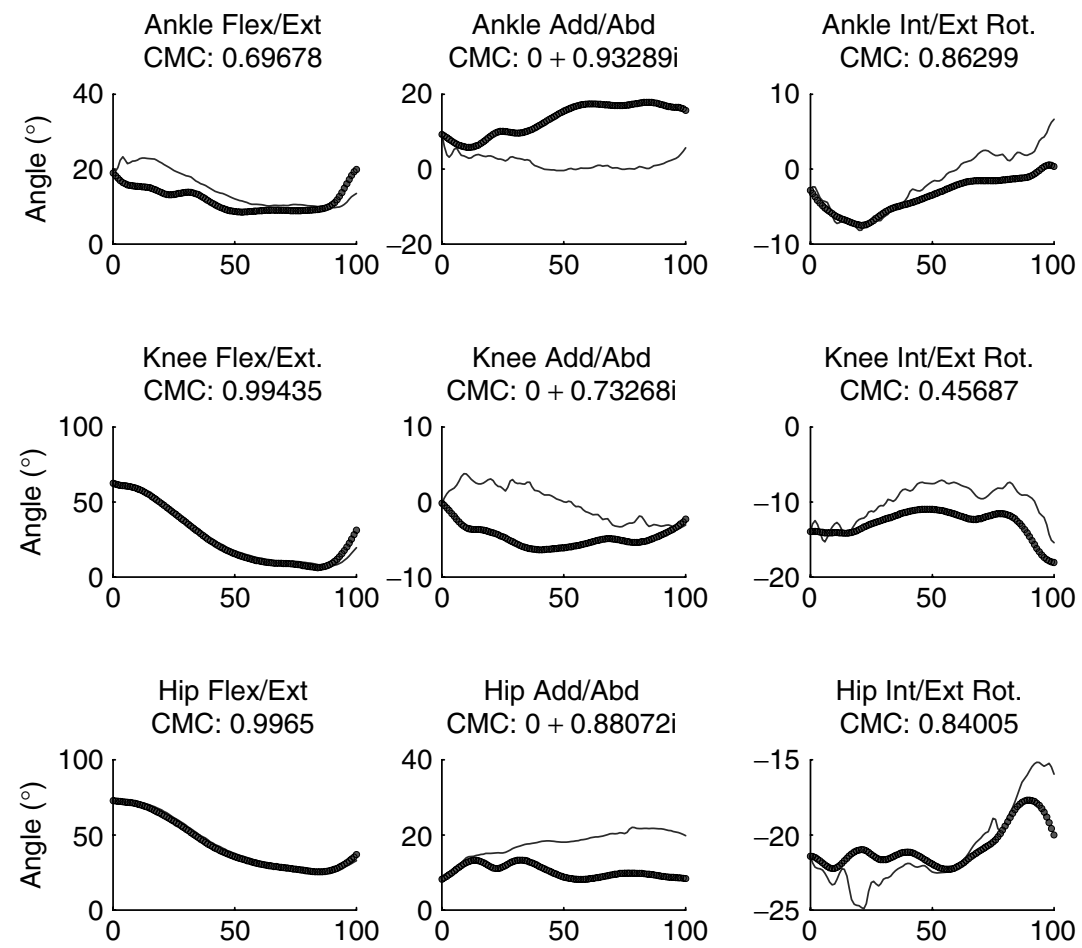

Figure 2. Sample kinematic data for a representative stroke subject performing an SOS descent trial (thicker lines represent IMU, and thin solid lines represent motion capture). Strong correlation observed for the estimated ankle, knee, and hip flexion/extension angles. 
Table 1. Mean range of motion for all joint angles of healthy and stroke subjects measured by motion capture

\begin{tabular}{|c|c|c|c|c|c|c|c|c|c|}
\hline \multirow{2}{*}{$\frac{\text { Subjects }}{\text { Joint angle }}$} & \multicolumn{3}{|c|}{ Healthy } & \multicolumn{3}{|c|}{ Affected } & \multicolumn{3}{|c|}{ Less affected } \\
\hline & FE & $\mathbf{A A}$ & IE & FE & AA & IE & FE & LA & IE \\
\hline Ankle & $53.28^{\circ}$ & $14.27^{\circ}$ & $13.63^{\circ}$ & $52.28^{\circ}$ & $18.26^{\circ}$ & $17.20^{\circ}$ & $36.86^{\circ}$ & $17.25^{\circ}$ & $15.61^{\circ}$ \\
\hline Knee & $69.52^{\circ}$ & $12.38^{\circ}$ & $14.03^{\circ}$ & $77.11^{\circ}$ & $13.48^{\circ}$ & $16.89^{\circ}$ & $68.05^{\circ}$ & $8.31^{\circ}$ & $13.62^{\circ}$ \\
\hline Hip & $20.37^{\circ}$ & $14.33^{\circ}$ & $16.47^{\circ}$ & $19.90^{\circ}$ & $13.30^{\circ}$ & $17.76^{\circ}$ & $17.80^{\circ}$ & $15.89^{\circ}$ & $17.81^{\circ}$ \\
\hline
\end{tabular}

The differences in RoM, grand mean error, SD, and SEM between joint-angles calculated through motion capture and IMUs are shown in Table 2 for the ankle, knee, and hip joints. Twenty-one trials for healthy limbs and 18 trials for both affected and less-affected limbs were included in this analysis (exclusions were due to partial motion capture marker occlusion that occurred in certain trials or the inability of certain stroke survivors to complete three successful trials of a given condition). On average, grand mean error values were lowest for all healthy joints in FE during both ascent and descent trials. At the ankle, offset correction was observed to have a significant effect on decreasing grand mean errors for all healthy, less affected and affected limbs $(p<0.01)$. The improvements in grand mean error values at the ankle observed in the affected and less affected limb trials for FE, AA, and IE rotation were as follows:

- $\quad$ FE grand mean error decreased from $14.1 \pm 5.3^{\circ}$ to $4.6 \pm 3.3^{\circ}(p=0.01)$ after offset correction for affected joints and from $7.2 \pm 3.5^{\circ}$ to $6.6 \pm 3.4^{\circ}(p=0.004)$ after offset correction for less affected joints.

- $\quad$ AA grand mean error decreased from $13.2 \pm 4.7^{\circ}$ to $4.2 \pm 3.2^{\circ}(p=0.01)$ after offset correction for affected joints and from $14.0 \pm 5.2^{\circ}$ to $4.8 \pm 3.3^{\circ}(p=1.12 \mathrm{E}-5)$ after offset correction for less affected joints.

- $\quad$ IE grand mean error decreased from $40.0 \pm 3.7^{\circ}$ to $2.8 \pm 2.4^{\circ}(\mathrm{p}=9.31 \mathrm{E}-6)$ after offset correction for affected joints and from $32.1 \pm 2.8^{\circ}$ to $2.2 \pm 1.7^{\circ}(p=0.03)$ after offset correction for less affected joints.

At the knee and hip joints, for both ascent and descent trials, improvement in grand mean error values were observed after offset correction in all limbs. The improvements in affected and less affected limb grand mean error values were as follows:

- $\quad$ At the knee joint, FE grand mean error decreased from $8.3 \pm 2.5^{\circ}$ to $2.1 \pm 1.7^{\circ}$ ( $p$ $=2.22 \mathrm{E}-6)$ and from $8.0 \pm 2.6^{\circ}$ to $2.3 \pm 1.9^{\circ}(p=1.1 \mathrm{E}-5)$ for affected and less affected limbs, respectively, after offset correction. At the hip joint, FE grand mean error decreased from $19.0 \pm 4.4^{\circ}$ to $4.0 \pm 2.3^{\circ}(p=0.08)$ and from $10.7 \pm$ $2.4^{\circ}$ to $2.1 \pm 1.5^{\circ}(p=0.01)$ for affected and less affected limbs respectively after offset correction.

- At the knee joint, AA grand mean error decreased from $11.9 \pm 4.6^{\circ}$ to $4.1 \pm 2.6^{\circ}$ ( $p$ $=0.001)$ and from $8.6 \pm 4.4^{\circ}$ to $4.0 \pm 3.0^{\circ}(p=0.001)$ for affected and less affected limbs, respectively, after offset correction. At the hip joint, AA grand mean error decreased from $5.1 \pm 2.0^{\circ}$ to $1.9 \pm 1.5^{\circ}(p=0.01)$ and from $8.3 \pm 2.9^{\circ}$ to $2.6 \pm 2.1^{\circ}$ ( $p=0.0001)$ for affected and less affected limbs respectively after offset correction. 

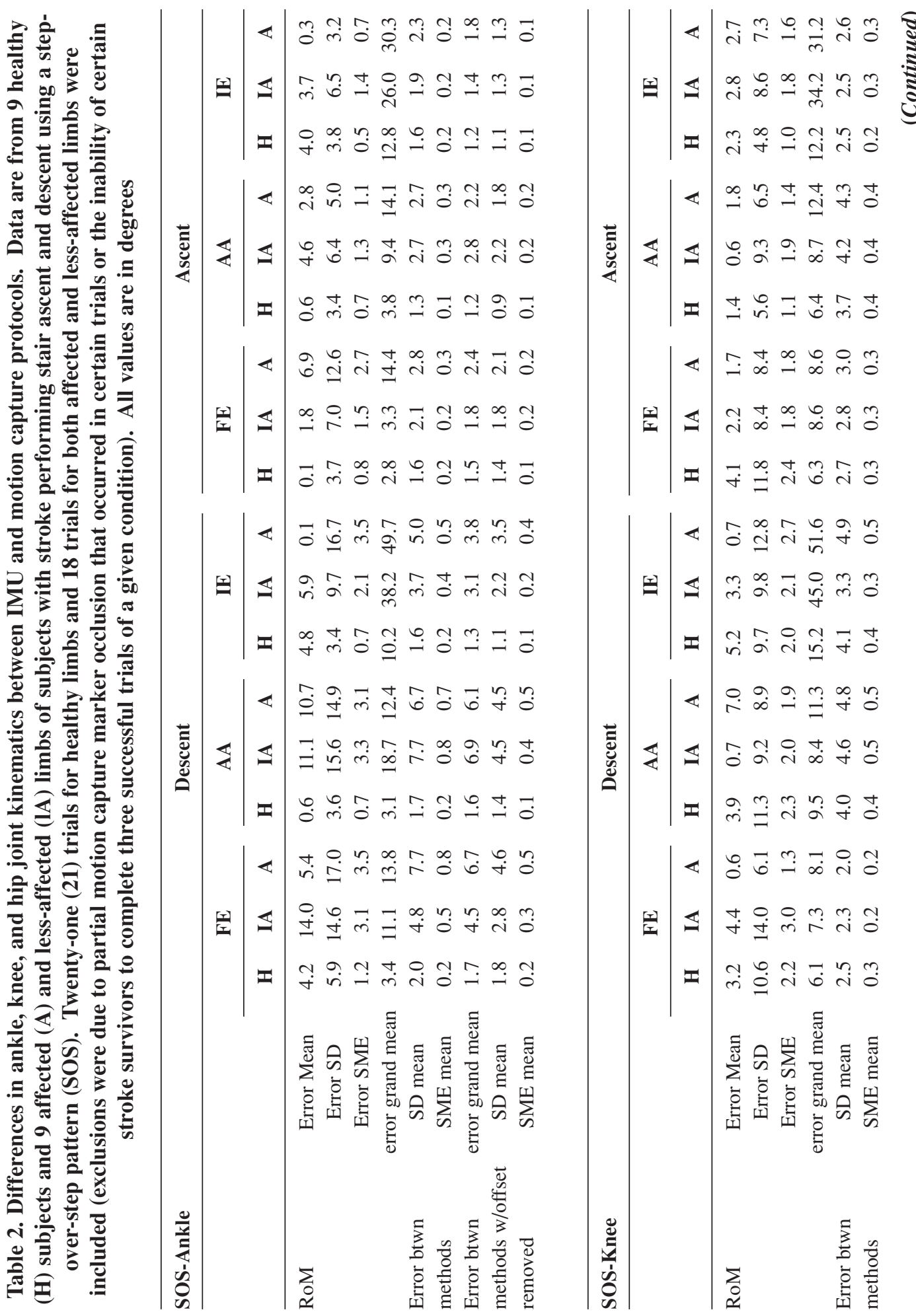

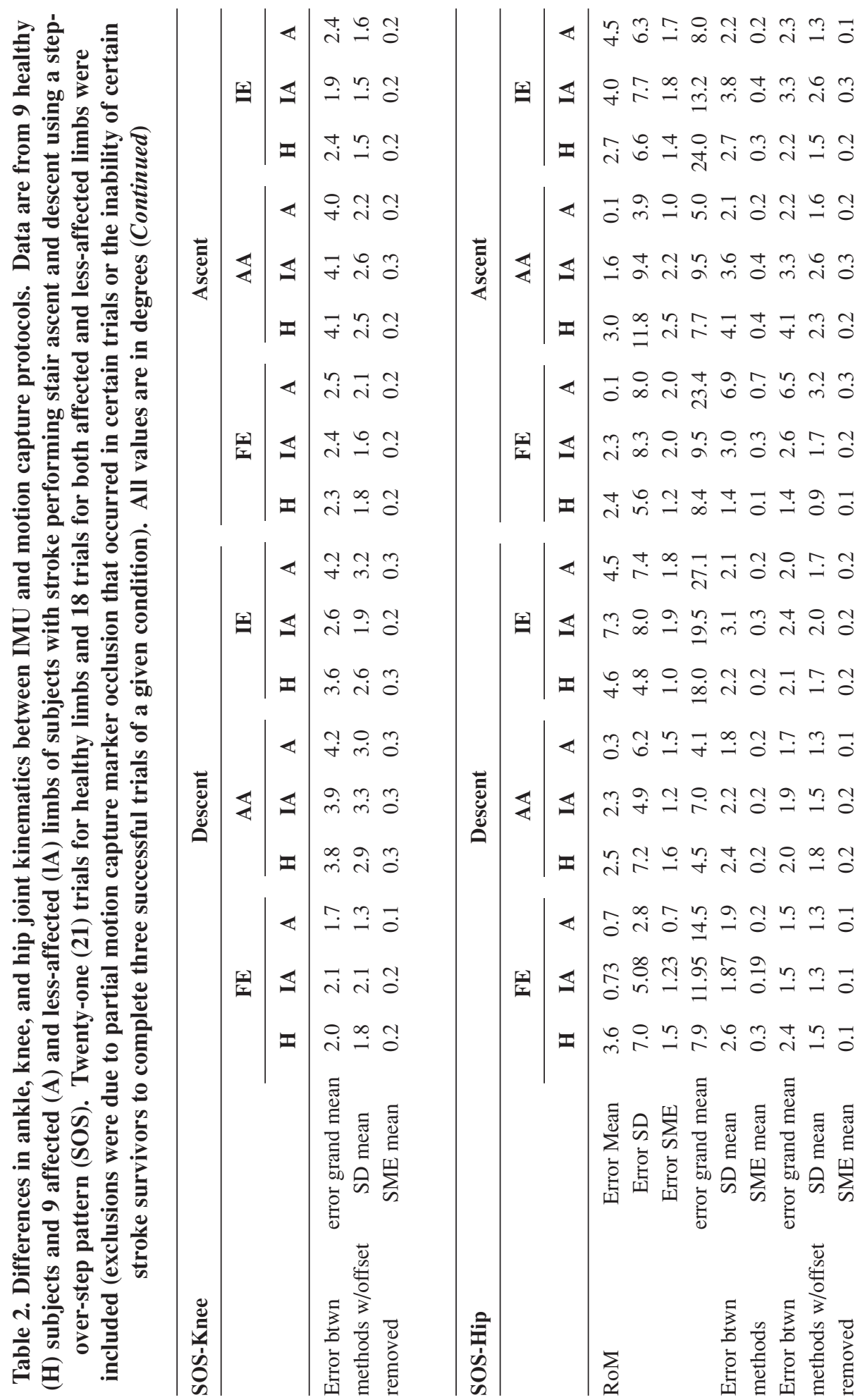
- At the knee joint, IE grand mean error decreased from $41.4 \pm 3.8^{\circ}$ to $3.3 \pm 2.4^{\circ}$ $(p=0.0007)$ and from $39.6 \pm 2.9^{\circ}$ to $2.3 \pm 1.7^{\circ}(p=0.05)$ for affected and less affected limbs, respectively, after offset correction. At the hip joint, IE grand mean error decreased from $17.6 \pm 2.2^{\circ}$ to $2.2 \pm 1.5^{\circ}(p=0.02)$ and from $16.3 \pm 3.5^{\circ}$ to $2.9 \pm 2.3^{\circ}(p=0.006)$ for affected and less affected limbs respectively after offset correction.

Mean differences in RoM values were calculated between protocols and were found to vary greatly between joints and joint angles. During ascent and descent trials, the following mean RoM error values were reported across joints:

- $\quad$ For healthy limbs: $2.9 \pm 7.4^{\circ}$ for $\mathrm{FE}, 2.0 \pm 7.1^{\circ}$ for $\mathrm{AA}$, and $3.9 \pm 5.5^{\circ}$ for IE rotation.

- $\quad$ For less affected limbs: $4.1 \pm 8.7^{\circ}$ for FE; $3.5 \pm 9.1^{\circ}$ for AA, and $4.5 \pm 8.4^{\circ}$ for IE rotation.

- $\quad$ For affected limbs: $2.6 \pm 9.2^{\circ}$ for $\mathrm{FE}, 3.8 \pm 7.6^{\circ}$ for $\mathrm{AA}$, and $2.1 \pm 9.0^{\circ}$ for IE rotation.

Standard deviations of mean RoM error values were found to be higher in both less affected and affected limbs than in healthy limbs.

Figures 3-5 report the $C M C_{1}$ and $C M C_{2}$ values for the ankle, knee, and hip joints respectively, for ascent and descent SOS H trials. Twenty-one trials for healthy limbs and 18 trials for both affected and less-affected limbs were included in this analysis. In each box-and-whisker plot, a comparison of the kinematics calculated through conventional laboratory based motion capture and IMU protocols is presented as one $C M C$ value per joint-angle for all healthy and stroke subjects. In Figures 3a-5a, $C M C_{1}$ box-and-whisker plots reflect joint-angles in which all non-real numbers have been removed. Real $C M C_{1}$ values were obtained for flexion/extension angles for both ascent and descent, with at least good similarity at the ankle and the knee joints and moderate similarity at the hip joint for both healthy and stroke subjects. For knee FE, values within whiskers for descent were greater than 0.87 (very good similarity), with median $C M C$ values above 0.94 . Values within whiskers for knee FE ascent trials were greater than 0.85 with medians above 0.90 (very good similarity).

After the offsets between corresponding motion capture and IMU waveforms were removed, $C M C$ values were recalculated as shown in Figures $3 \mathrm{~b}-5 \mathrm{~b} . C M C_{2} \mathrm{FE}$ median values were at least 0.91 for all joints, suggesting very good similarity. Notably, all knee FE values within whiskers for ascent conditions were above 0.97, and those for descent conditions were above 0.94, suggesting very good and excellent similarity, respectively. However, abduction/adduction and internal/external rotation angle values, even after the offset was corrected for, fell below the acceptable range of $C M C$ values. Therefore, the strongest correlation between protocols was observed between calculations of flexion/extension angles at the ankle, knee, and hip joints. The highest levels of correlation between protocols were found in the calculation of healthy joint angles, while for stroke subjects, correlation was found to be weaker in the less affected and affected limbs, suggesting that IMUs estimated joint angles for healthy older adults more accurately than for stroke subjects. 
(a)

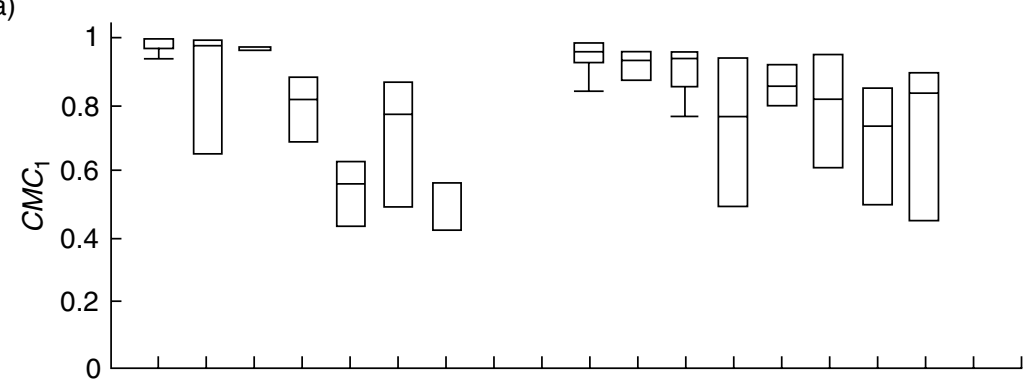

(b)

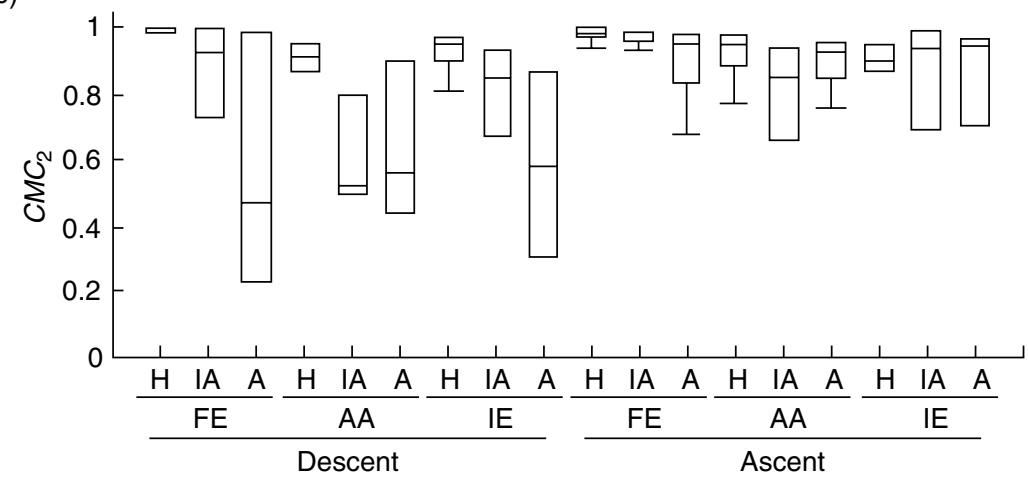

Figure 3. Box-and-whiskers plots for $C M C_{1}$ (a) and $C M C_{2}$ (b) at the ankle joint. $C M C_{1}$ and $C M C_{2}$ measure the within joint-angle similarity between IMU and motion capture waveforms. Distributions reflect 21 normal healthy $(\mathrm{H})$ trials, and 18 affected $(\mathrm{A})$ and less-affected (1A) trials for flexion/extension (FE), abduction/adduction (AA) and internal/external rotation (IE) angles. Box-plots are reported only for those joint-angles in which all values were real numbers.

\section{DISCUSSION}

In this study, we aimed to perform a validation of the feasibility of using Xsens IMUs in the gait analysis of chronic stroke survivors as well as healthy older adults during stair ambulation. Ten chronic stroke survivors and ten healthy older adults were recruited for this study, and 9 from each group were included in the analysis in order to observe stair ambulation techniques relative to various degrees of mobility impairments. The application of IMUs in gait analysis of this kind will be possible only if the detection of gait abnormalities in joint kinematics through this technology can be proven similar to conventional laboratory-based motion capture systems. If demonstrated, the use of these 9D IMU sensors could be extended to study different populations outside laboratories in day-to-day settings while remaining minimally invasive. Comparing data simultaneously collected through conventional motion 
(a)

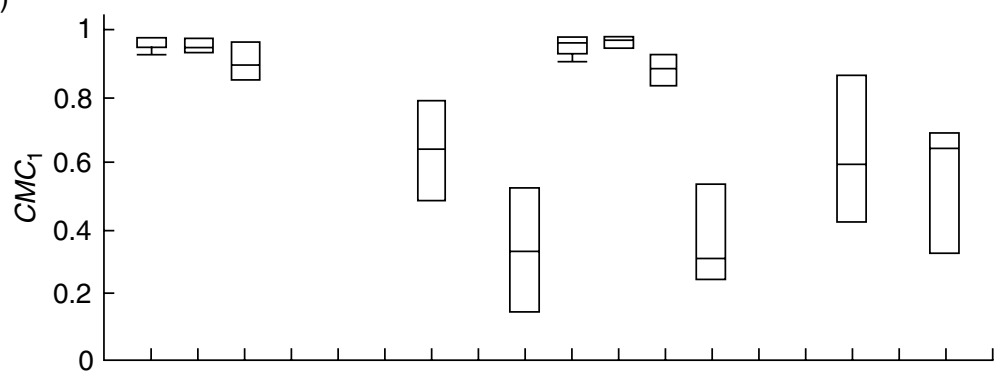

(b)

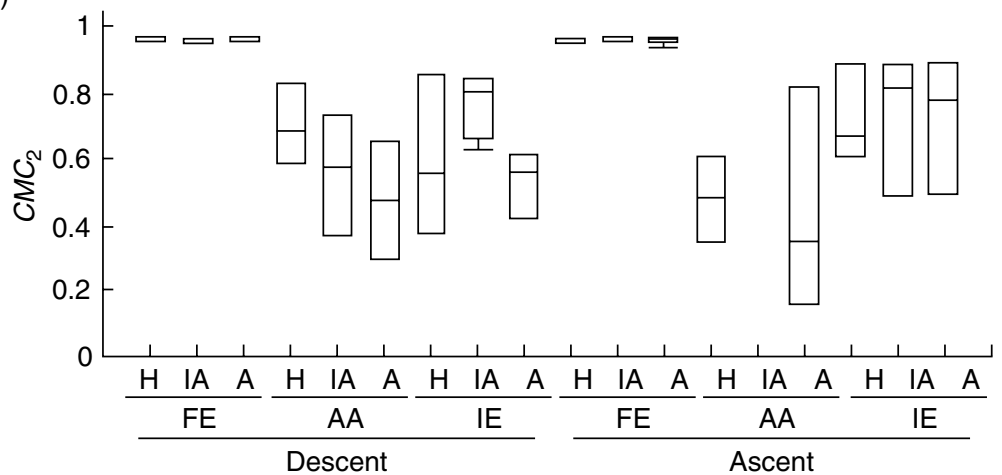

Figure 4. Box-and-whiskers plots for $C M C_{1}$ (a) and $C M C_{2}$ (b) at the knee joint. $C M C_{1}$ and $C M C_{2}$ measure the within joint-angle similarity between IMU and motion capture waveforms. Distributions reflect 21 normal healthy (H) trials, and 18 affected (A) and less-affected (1A) trials for flexion/extension (FE), abduction/adduction (AA) and internal/external rotation (IE) angles. Box-plots are reported only for those joint-angles in which all values were real numbers.

capture and through IMUs calibrated using the sensors' proprietary software, it was found that similar to previous studies in healthy young subjects by Ferrari et al. [16] and van den Noort et al. [26], differences between sensors were largest for the transverse and frontal plane angles (abduction/adduction and internal/external rotation). Differences in kinematics between IMU and motion capture methods were found to be higher in those subjects with stroke than in the healthy older adults, yet differences in all three conditions were found to be greater than those reported by Ferrari et al. and by van den Noort et al. using the Outwalk protocol $[16,26]$. The greater differences between previous studies and this work however can likely be attributed to differences in age and associated adaptations in locomotion patterns of individuals in this study compared with the young healthy population previously studied [32]. In addition, differences can also be attributed to the various calibration 
(a)

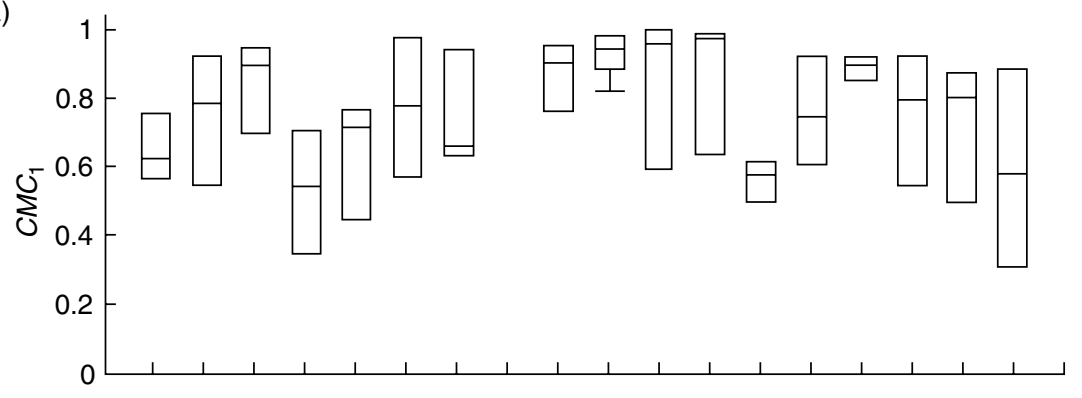

(b)

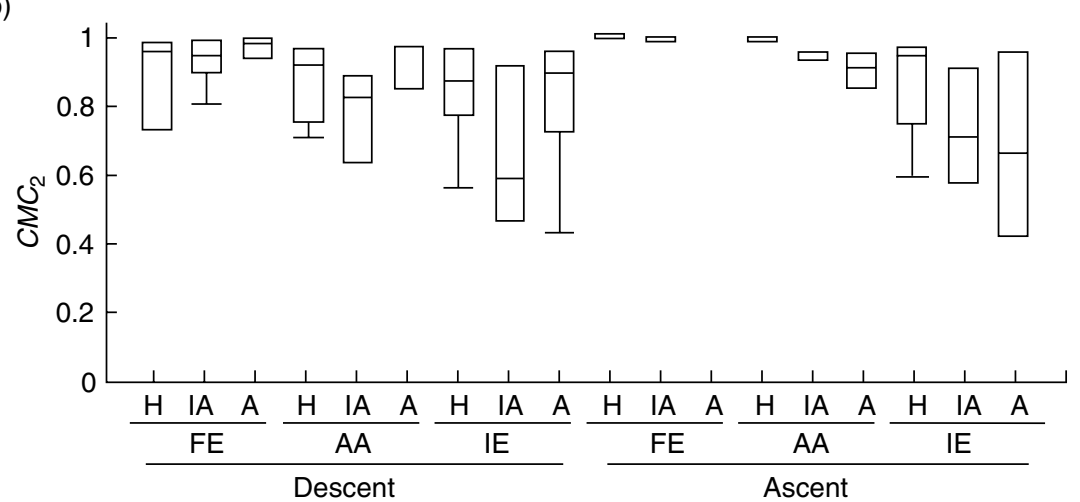

Figure 5. Box-and-whiskers plots for $C M C_{1}$ (a) and $C M C_{2}$ (b) at the hip joint. $C M C_{1}$ and $C M C_{2}$ measure the within joint-angle similarity between IMU and motion capture waveforms. Distributions reflect 21 normal healthy (H) trials, and 18 affected (A) and less-affected (1A) trials for flexion/extension (FE), abduction/adduction (AA) and internal/external rotation (IE) angles. Box-plots are reported only for those joint-angles in which all values were real numbers.

procedures used in previous studies compared with the static calibration proposed in this study. For both stroke and healthy older populations, flexion extension angles (sagittal plane) reported at the ankle, knee, and hip proved to yield the highest levels of similarity between motion capture and inertial sensor protocols, as reported through $C M C_{1}$ values. This similarity can be attributed in part to the greater amplitude in flexion extension angles, hence resulting in a much greater signal-tonoise ratio than present in the remaining joint angles. Therefore, it can be concluded that 9D IMU sensors with upright calibration performs with moderate accuracy, without offset removal, in the estimation of joint-angle in the sagittal plane. Additionally, after removing the offset between corresponding waveforms, the $\mathrm{CMC}_{2}$ values for these joint-angles were found to improve to very good similarity. 
Therefore, when using only static upright calibration for the IMU-based system, offset correction or calibration with a reference camera-based system may prove necessary in order to obtain accurate estimates of joint angle in individuals having suffered a stroke. The results of the current comparison may not apply to other IMU systems if they use different protocols in joint angle estimation.

The quality of similarity of joint-angle estimation between protocols shown through $C M C_{2}$ values for ascent and descent in the SOS condition is similar to those in the SBS condition. The portability of IMUs could afford the ease of data collection within a natural and more familiar environment, which would be particularly valuable for assessing mobility in people with impairments (such as hemiparesis due to stroke) and developing gait retraining rehabilitation interventions.

The improvement in $C M C$ results after offset correction suggests a potential means of using inertial sensors for the measurement of joint angles during stair ambulation in post-stroke subjects outside traditional laboratory settings. It is possible to calibrate the inertial sensor system with a camera-based motion capture system by collecting a series of stair ambulation data within a laboratory. From these trials, mean offset values between systems could be established and used to correct subsequent inertial data collected during non-laboratory stair ambulation activities.

After offset correction, certain calculated $C M C_{2}$ values were found to have decreased from $C M C_{1}$ values calculated using raw data. This decrease can be attributed to a greater number of non-imaginary $C M C$ values affecting the mean $C M C$ for each limb. The calculation of $C M C$ values may also be affected by the differences in RoM observed between affected and less-affected limbs. Greater RoM results in a greater $\bar{Y}_{g}$ value in eqn. 1 , which in turn would result in a greater $C M C$ value. Therefore, $C M C$ may not be the best measure of correlation between curves in populations with mobility impairment, where a greater range of mobility may be experienced in less affected limbs [41].

A higher correspondence in joint angle estimation between protocols for healthy subjects was demonstrated through RoM, grand mean error, SD and SEM results under both ascent and descent conditions, where the strongest similarities were observed for the sagittal plane angles. However, for subjects with chronic stroke, a greater difference in the estimation of joint angles through the IMU and motion capture protocols was found. The error in joint angle estimation for stroke patients was further found to lie beyond the limits reported by Yavuzer et al. and Novak et al. [30-32]. The differences in joint angles reported, primarily within the frontal and transverse planes, are believed to be the result of the calibration procedures used in each protocol to define the local CS of the foot, shank, and thigh. The effect of anatomical frame variation on joint angles was described by Brennan et al. [42] such that any perturbation of the nominal anatomical frame definition would result in kinematic crosstalk.

For those angles in the sagittal plane for both healthy and stroke groups, mean RoM values reported were found to lie just beyond the range of $\pm 5^{\circ}$ reported using the Outwalk protocol involving healthy adults [29]. The increase can be attributed to IMU accuracy and calibration procedures, yet these RoM values for FE angles suggest that Xsens IMU sensors could reliably be used for the measurement of primary joint angles in stroke patients. For rehabilitation aiming at restoring or maintaining RoM of affected 
limbs, IMUs could serve as an important tool in the evaluative measure of recovery from hemiplegic stroke [43, 44].

In subjects with chronic stroke, the differences in estimated joint angles between protocols may be due to the differences in local CSs definitions. The calibration of the Xsens IMUs requires only that participants maintain a neutral stance position so that the orientation and distance of each IMU can be determined. However, this calibration procedure functions under the assumption that the subjects body segments are aligned so as to maintain the nominal joint-angles of the neutral stance position during the calibration procedure. Yet, the ability of a healthy individual to comply with these requirements is far easier than that of an individual with stroke, who may present less stability and lower limb strength. Chronic muscle weakness may also play a role in the inability to achieve an accurate anatomical calibration solely from a static posture in stroke survivors. Additional calibration procedures should therefore be developed, as was done for the Outwalk protocol applied to children with cerebral palsy [16], allowing for repeatable and reliable measurements to be acquired through IMU sensors outside laboratory settings. The validation of IMUs in the assessment of gait in impaired populations through Outwalk suggests that perhaps activity- or population-specific calibration of these sensors may be required. A more specialized functional calibration for chronic stroke survivors might result in a more accurate definition of joint axes and sensor position rather than simply relying on the definition of axes through bony landmarks and the recording of a static pose.

Additional sources of error present in this study may include rater reliability in identifying bony landmarks during motion capture calibration, determination of heelstrike and toe-off gait events based on IMU kinematic data, different sampling frequencies between systems, and normalization of data to 101 points for the description of stance phase in both IMU and motion capture systems. Any one of these sources of error in isolation or combination could contribute to the differences in estimated joint angles observed between systems.

A limitation of this study is that only the joint angles during stance phase were compared due to partial IRED marker occlusion that occurred during the late swing phase. Marker occlusion is a challenge associated with motion capture systems due to the line of sight requirements between cameras and markers, an issue that does not impact IMU sensors. Given that the stance phase represents the majority of the overall stride time ( $64 \%$ of the stride cycle), this limitation is considered not serious. It is also the case that in negotiating stairs, a major point of instability during stance is when the total body weight is transferred from one limb to the other while all joints are flexed [45]. Previous work in stroke and stair ambulation have focused on understanding and characterizing the stance phase [6]. The inclusion of swing phase in comparison studies would enable determination of whether the differences in acceleration profiles in stance and swing could impact the accuracy of the IMU data.

A second limitation of this study is that the tests were not conducted in a random order. Throughout the course of data collection, subjects might have become more familiar with the testing environment and the stepping patterns required, which might have affected stepping speed, ease of motion, and joint angles during trials (i.e., the learning effect). Had stepping conditions been randomized throughout testing, the learning effect may have 
been minimized. However, we believe that if learning effect was a factor in this study, it would have equally affected all stepping conditions and groups.

\section{CONCLUSIONS}

In this study, the application of IMUs was found to be suitable for the estimation of major joint angles in healthy older populations. In addition, for clinical research and rehabilitation, IMU sensors could be employed in the analysis of dynamic RoM in stroke survivors. However, further research is needed before IMUs can be applied to estimate frontal and transversal plane angles in both elderly and stroke populations. To extend this technology to the estimation of major joint angles in stroke patients will require further research to establish a new calibration procedure better suited for these individuals in order to provide valid data; i.e. to reduce the discrepancies between measured joint-angles and those obtained through conventional motion capture protocols. With an improved calibration procedure for the IMU protocol, this technology can extend gait and stair ambulation evaluation for stroke patients to a nonlaboratory, real-world environment.

\section{ACKNOWLEDGEMENTS}

This work was partially supported by an NSERC grant to Q.L. and an HSF grant to B.B. We also would like to thank Heather Ridgway for assistance in data collection.

\section{CONFLICT OF INTEREST}

The authors indicated no potential conflicts of interest.

\section{REFERENCES}

[1] J. K. Startzell, D. A. Owens, L. M. Mulfinger, and P. R. Cavanagh, "Stair negotiation in older people/: A review," Journal of the American Geriatrics Society, 2000, 48(5):567-580.

[2] M. Oh-Park, S. Perera, and J. Verghese, "Clinically meaningful change in stair negotiation performance in older adults.," Gait \& posture, 2012, 36 (3):532-5366.

[3] A. Protopapadaki, W. I. Drechsler, M. C. Cramp, F. J. Coutts, and O. M. Scott, "Hip, knee, ankle kinematics and kinetics during stair ascent and descent in healthy young individuals.," Clinical biomechanics (Bristol, Avon), 2007, 22(2):203-210.

[4] S. Nadeau, B. . McFadyen, and F. Malouin, "Frontal and sagittal plane analyses of the stair climbing task in healthy adults aged over 40 years: what are the challenges compared to level walking?," Clinical Biomechanics, 2003, 18(10):950-959.

[5] A. C. Novak, Q. Li, S. Yang, and B. Brouwer, "Mechanical energy transfers across lower limb segments during stair ascent and descent in young and healthy older adults.," Gait \& posture, 2011, 34(3):384-390.

[6] A. C. Novak, "Biomechanical and physical requirements of stair negotiation with respect to aging and stroke," Ph.D. dissertation, Queen's University, 2011.

[7] L. L. Ploutz-Snyder, T. Manini, R. J. Ploutz-Snyder, and D. a Wolf, "Functionally relevant thresholds of quadriceps femoris strength.," The journals of gerontology. Series A, Biological sciences and medical sciences, 2002, 57(4):144-152.

[8] G. J. Salem, M. Y. Wang, J. T. Young, M. Marion, and G. a Greendale, "Knee strength and lower- and higher-intensity functional performance in older adults.," Medicine and science in sports and exercise, 2000, 32(10):1679-1684.

[9] J. Perry, M. Garrett, J. K. Gronley, and S. J. Mulroy, "Classification of Walking Handicap in the Stroke Population," Stroke, 1995, 26(6):982-989. 
[10] S. E. Lord, K. McPherson, H. K. McNaughton, L. Rochester, and M. Weatherall, "Community ambulation after stroke: how important and obtainable is it and what measures appear predictive?," Archives of Physical Medicine and Rehabilitation, 2004, 85(2):234-239.

[11] R. B. Guralnik, J.M.; Simonsick, E.M.; Ferrucci, L.; Glynn, R.J.; Berkman, L.F.; Blazer, D.G.; Scherr, P.A.; Wallace, "A Short physical performance battery assessing lower extremity function: association with self-reported disability and prediction of mortality and nursing home admission," Journal of Gerontology, 1994, 49(2):85-94.

[12] C. Bonnyaud, R. Zory, D. Pradon, N. Vuillerme, and N. Roche, "Clinical and biomechanical factors which predict Timed Up and Down Stairs test performance in hemiparetic patients.," Gait \& posture, 2013, 38(3):466-470.

[13] M. Alzahrani, C. Dean, and L. Ada, "Relationship between walking performance and types of community-based activities in people with stroke: an observational study.," Revista brasileira de fisioterapia (São Carlos (São Paulo, Brazil)), 2011, 15(1):45-51.

[14] M. A. Alzahrani, C. M. Dean, and L. Ada, "Ability to negotiate stairs predicts free-living physical activity in community-dwelling people with stroke: an observational study.," The Australian journal of physiotherapy, 2009, 55(4):277-281.

[15] M. Thorngren, B. Westling, and B. Norrving, "Outcome after stroke in patients discharged to independent living," Stroke, 1990, 21(2):236-240.

[16] A. G. Cutti, A. Ferrari, P. Garofalo, M. Raggi, A. Cappello, and A. Ferrari, “'Outwalk’: a protocol for clinical gait analysis based on inertial and magnetic sensors.," Medical \& biological engineering \& computing, 2010, 48(1):17-25.

[17] H. M. Schepers, H. F. J. M. Koopman, and P. H. Veltink, "Ambulatory assessment of ankle and foot dynamics.," IEEE transactions on bio-medical engineering, 2007, 54(5):895-902.

[18] H. Martin Schepers, E. H. F. van Asseldonk, C. T. M. Baten, and P. H. Veltink, "Ambulatory estimation of foot placement during walking using inertial sensors.," Journal of biomechanics, 2010, 43(16):3138-3143.

[19] D. Roetenberg, "Inertial and Magnetic Sensing of Human Motion,", Ph.D. disertation , Univeristy of Twente, 2006.

[20] A. M. Sabatini, "Quaternion-based extended Kalman filter for determining orientation by inertial and magnetic sensing.," IEEE transactions on bio-medical engineering, 2006, 53(7):1346-1356.

[21] T. Liu, Y. Inoue, K. Shibata, K. Shiojima, and J. Bin Yin, “A Novel Three-Dimensional Gait Analysis System," Advanced Materials Research, 2012, 569:352-355.

[22] S. Lambrecht, I. Jonkers, and J. L. Pons, "Converging Clinical and Engineering Research on Neurorehabilitation,", Vol.1, Springer, 2013.

[23] K. J. O’Donovan, R. Kamnik, D. T. O’Keeffe, and G. M. Lyons, "An inertial and magnetic sensor based technique for joint angle measurement.," Journal of biomechanics, 2007, 40(12):2604-2611.

[24] P. Picerno, A. Cereatti, and A. Cappozzo, "Joint kinematics estimate using wearable inertial and magnetic sensing modules.," Gait \& posture, 2008, 28(4):588-595.

[25] H. Zhou, H. Hu, and N. Harris, "Application of wearable inertial sensors in stroke rehabilitation.," Conference proceedings/IEEE Engineering in Medicine and Biology Society, 2005, 4:6825-6828.

[26] J. C. van den Noort, A. Ferrari, A. G. Cutti, J. G. Becher, and J. Harlaar, "Gait analysis in children with cerebral palsy via inertial and magnetic sensors.," Medical \& biological engineering \& computing, 2013, 51(4):377-386.

[27] S. Yang, J.-T. Zhang, A. C. Novak, B. Brouwer, and Q. Li, "Estimation of spatio-temporal parameters for post-stroke hemiparetic gait using inertial sensors.," Gait \& posture, 2013, 37(3):354-358.

[28] A. S. Anna, N. Wickstrom, R. Zugner and R. Tranberg, "A wearable gait analysis system using inertial sensors Part I/: Evaluation of measures of gait symmetry and normality against 3D kinematic data," Biosignals, 2012:180-188.

[29] A. Ferrari, A. G. Cutti, P. Garofalo, M. Raggi, M. Heijboer, A. Cappello, and A. Davalli, "First in vivo assessment of 'Outwalk': a novel protocol for clinical gait analysis based on inertial and magnetic sensors.," Medical \& biological engineering \& computing, 2010, 48(1):1-15. 
[30] G. Yavuzer, O. Oken, A. Elhan, and H. J. Stam, "Repeatability of lower limb three-dimensional kinematics in patients with stroke.," Gait \& posture, 2008, 27(1):31-35.

[31] A. C. Novak, "The effect of botulinum toxin a (BTX-A) on gait in chronic stroke," MA. Sc. thesis, Queen's University, 2007.

[32] A. C. Novak and B. Brouwer, "Kinematic and Kinetic Evaluation of the Stance Phase of Stair Ambulation in Persons with Stroke and Healthy Adults: A Pilot Study," Journal of applied biomechanics, 2013, 29(4):443-452.

[33] T. Cloete and C. Scheffer, "Repeatability of an off-the-shelf, full body inertial motion capture system during clinical gait analysis.," Conference proceedings/IEEE Engineering in Medicine and Biology Society. Conference, 2010:5125-128.

[34] D. Roetenberg, H. Luinge, and P. Slycke, "Xsens MVN/: Full 6DOF Human Motion Tracking Using Miniature Inertial Sensors," Xsens Technologies white paper, pp. 1-7, 2009.

[35] A. C. Novak and B. Brouwer, "Sagittal and frontal lower limb joint moments during stair ascent and descent in young and older adults.," Gait \& posture, 2011, 33(1):54-60.

[36] B. Brouwer, K. Parvataneni, and S. J. Olney, "A comparison of gait biomechanics and metabolic requirements of overground and treadmill walking in people with stroke.," Clinical biomechanics (Bristol, Avon), 2009, 24(9):729-734.

[37] D. A. Winter, Biomechanics and Motor Control of Human Movement. Wiley, 2009.

[38] M. P. Kadaba, H. K. Ramakrishnan, M. E. Wootten, J. Gainey, G. Gorton, and G. V Cochran, "Repeatability of kinematic, kinetic, and electromyographic data in normal adult gait.," Journal of orthopaedic research, 1989, _ 7(6):849-60.

[39] A. Ferrari, A. G. Cutti, and A. Cappello, "A new formulation of the coefficient of multiple correlation to assess the similarity of waveforms measured synchronously by different motion analysis protocols.," Gait \& posture, 2010, 31(4):540-542.

[40] P. Garofalo, A. G. Cutti, M. V. Filippi, S. Cavazza, A. Ferrari, A. Cappello, and A. Davalli, "Interoperator reliability and prediction bands of a novel protocol to measure the coordinated movements of shoulder-girdle and humerus in clinical settings.," Medical \& biological engineering \& computing, 2009, 47(5):475-486.

[41] J. Røislien, O. Skare, a Opheim, and L. Rennie, "Evaluating the properties of the coefficient of multiple correlation $(C M C)$ for kinematic gait data.," Journal of biomechanics, 2012, 45(11):2014-2018.

[42] A. Brennan, K. Deluzio, and Q. Li, "Assessment of anatomical frame variation effect on joint angles: A linear perturbation approach.," Journal of biomechanics, 2011, 44(16): 2838-2842.

[43] "Moving forward after a stroke.," The Johns Hopkins medical letter health after 50, vol. 2006, 17(12):4-5.

[44] D. J. Gladstone, C. J. Danells, and S. E. Black, "The Fugl-Meyer Assessment of Motor Recovery after Stroke: A Critical Review of Its Measurement Properties," Neurorehabilitation and Neural Repair, 2002, 16(3):232-240.

[45] B. J. McFadyen and D. a Winter, "An integrated biomechanical analysis of normal stair ascent and descent.," Journal of biomechanics, 1988, 21(9):733-744. 



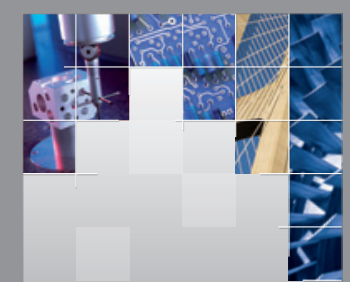

\section{Enfincering}
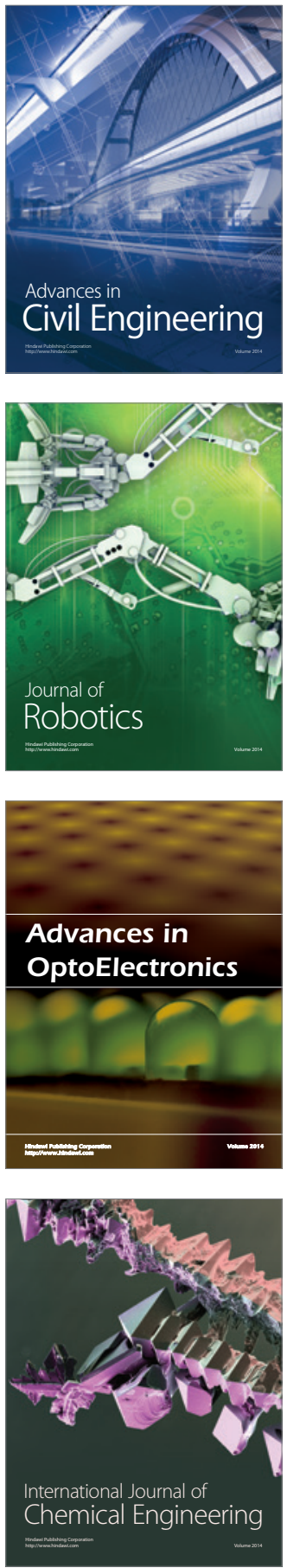

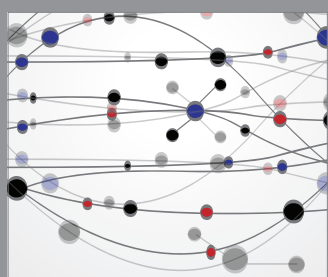

The Scientific World Journal

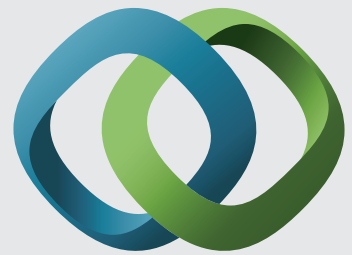

\section{Hindawi}

Submit your manuscripts at

http://www.hindawi.com
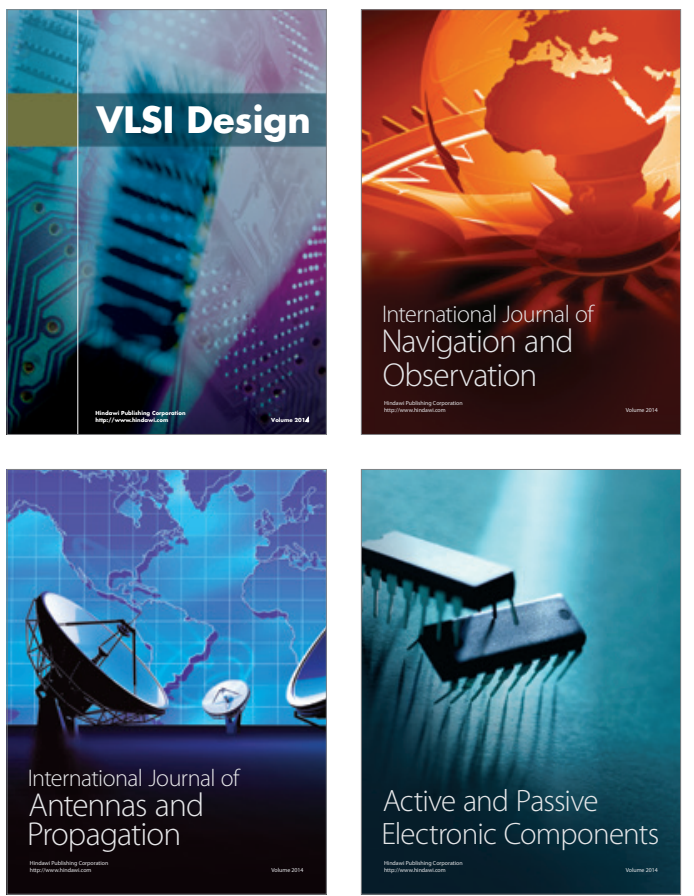
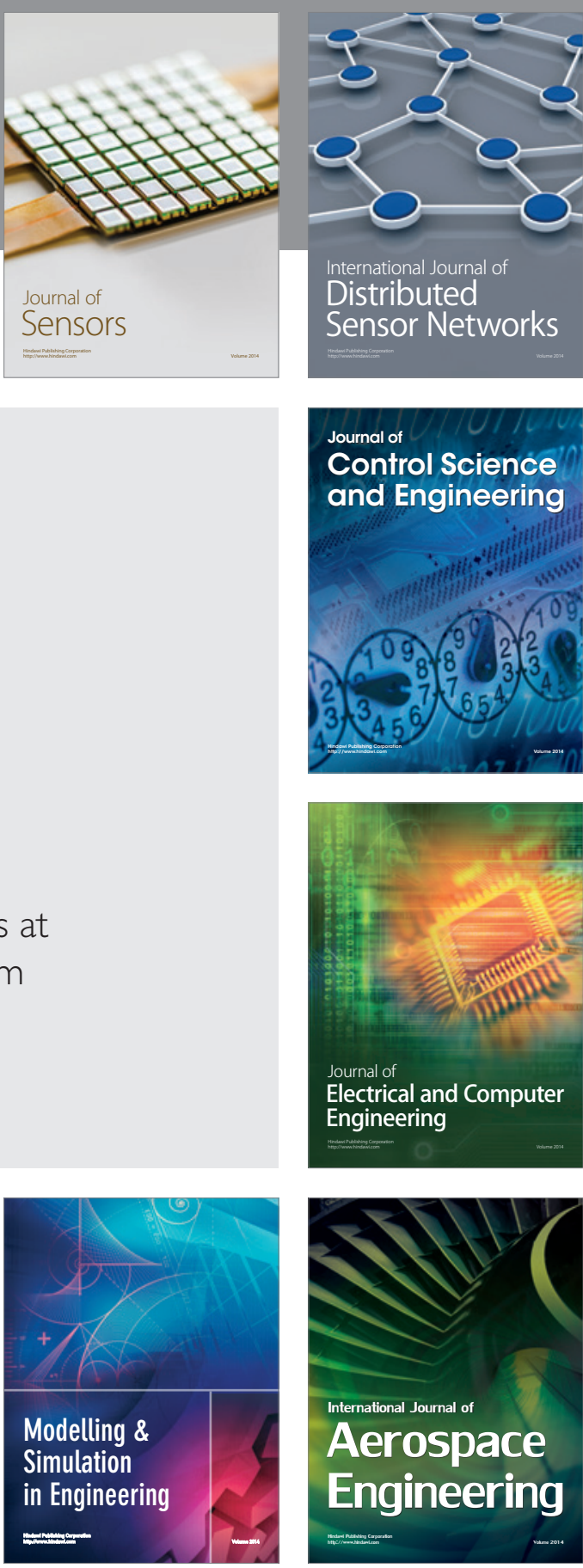

International Journal of

Distributed

Sensor Networks

Journal of

Control Science

and Engineering
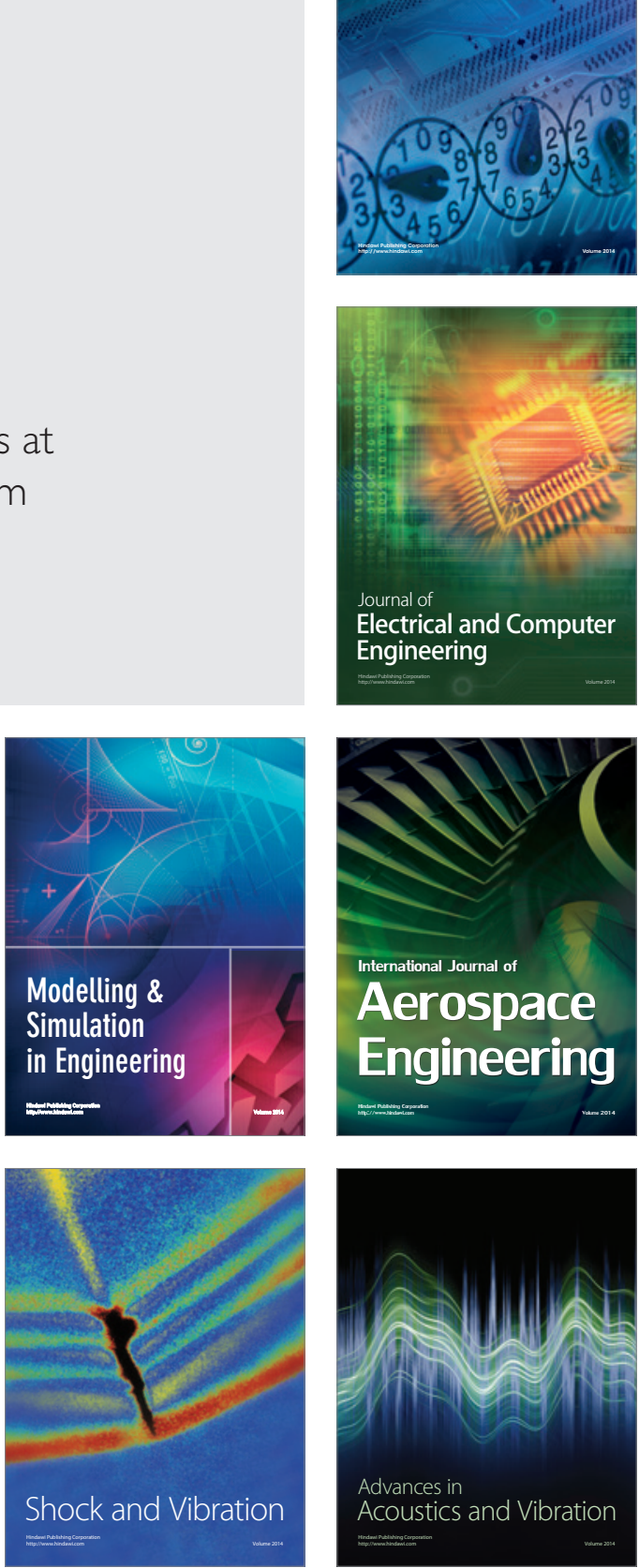